\title{
Pharmacological treatment of COPD: the devil is always in the detail
}

\author{
Dave Singh ${ }^{1}$, Peter J. Barnes ${ }^{2}$, Robert Stockley ${ }^{3}$, Maria Victorina Lopez Valera ${ }^{4}$, \\ Claus Vogelmeier ${ }^{5}$ and Alvar Agusti ${ }^{6}$
}

Affiliations: ${ }^{1}$ University of Manchester, Medicines Evaluation Unit, Manchester University NHS Foundation Hospital Trust, Manchester, UK. ${ }^{2}$ National Heart and Lung Institute, University of London Imperial College, London, UK. ${ }^{3}$ Lung Investigation Unit, University Hospital Birmingham, Birmingham, UK. ${ }^{4}$ Dept of Pulmonology, Universidad de la Republica Montevideo, Montevideo, Uruguay. ${ }^{5}$ Dept of Medicine, Pulmonary and Critical Care Medicine, University Medical Center Giessen and Marburg. Philipps-University Marburg. Member of the German Center for Lung Research (DZL), Marburg, Germany. ${ }^{6}$ Hospital Clínic, Universitat de Barcelona, Ciberes, Barcelona, Spain.

Correspondence: Dave Singh, University of Manchester, Medicines Evaluation Unit, The Langley Building, Manchester University NHS Foundation Hospital Trust, Southmoor Road, M23 9QZ, Manchester, UK.

E-mail: dsinghameu.org.uk

@ERSpublications

GOLD ABCD should be used for initial assessment and treatment, but follow-up treatment should focus on treatable traits http://ow.ly/Vm5130iXYYU

Cite this article as: Singh D, Barnes PJ, Stockley R, et al. Pharmacological treatment of COPD: the devil is always in the detail. Eur Respir J 2018; 51: 1800263 [https://doi.org/10.1183/13993003.00263-2018].

\section{The problem}

Chronic obstructive pulmonary disease (COPD) is a heterogeneous condition with considerable pathophysiological and clinical variability across patients [1]. For this reason, the current Global Initiative for the Management of Chronic Obstructive Lung Disease (GOLD) report recommends an individualised treatment approach for patients with a diagnosis of COPD that has been established by appropriate clinical and spirometric criteria [2]. This approach is based on symptom severity and exacerbation history (which predicts future exacerbation risk) [2], and categorises patients into four "phenotypic" groups (A, B, C or D) $[2,3]$. Each group has distinct pharmacological treatment algorithms where the clinical response to treatment (improvement, no change or deterioration) is used to escalate, de-escalate or switch treatment. It is straightforward in treatment-naïve individuals seen at initial presentation by healthcare workers but it may become more complex and cumbersome during follow-up.

GOLD recognises that these algorithms have varying levels of supporting evidence and that some of the current choices can be debated. Furthermore, since the devil is always in the detail, clinicians using these follow-up recommendations in clinical practice soon became aware of potential scenarios that deserve more careful thought and discussion. Below, we discuss some examples of the difficulties encountered in clinical practice during follow-up when trying to use the GOLD ABCD classification system. We propose as an alternative that the $A B C D$ system approach should be reserved for initiating pharmacological treatment, whereas treatment during follow-up should be focused on exacerbations and COPD symptoms such as dyspnoea, which are "treatable traits" $[4,5]$, without the need to refer to the $\mathrm{ABCD}$ system. A treatable trait is a therapeutic target identified by "phenotype" characteristics or "endotype" recognition through validated biomarker(s) $[4,5]$. While many treatable traits have been identified in COPD, we propose that targeting exacerbations and symptoms (specifically dyspnoea) while managing COPD patients during follow-up offers a personalised and straightforward approach. 


\section{Scenario 1}

The GOLD ABCD classification is intended to be carried out at initial presentation, when a patient is not receiving any relevant pharmacological treatment. Whether (and how) it should also be used in patients whose GOLD classification had not been previously documented but where patients are already receiving maintenance pharmacological treatment (i.e. long-acting bronchodilators, with or without inhaled corticosteroids (ICS)) is not explicitly clarified in the current GOLD report.

Applying the GOLD ABCD classification for the first time in patients already receiving pharmacological treatment implies an intention to subsequently apply the associated treatment algorithm. However, there is a flaw with this approach, particularly when considering optimising treatment for exacerbation prevention. Some patients might have previously received treatment(s) that led to clinical improvement and a reduction in exacerbations. This positive treatment response may assign the patient into a lower risk GOLD group ( $A$ or $B$ ) compared to that before treatment ( $C$ or $D)$. Using the lower risk GOLD classification reflecting the treatment response (A or B) may suggest the patient is over treated leading to a de-escalation pathway and hence withdrawal of a successful treatment, which is illogical.

It is important to remember that COPD patients usually have persistent symptoms even on effective therapy, which may have led to over-treatment despite no clear further benefit. This is particularly true with ICS which are licensed for use in patients with recurrent exacerbations, but are often used in clinical practice in patients who have not experienced such events. Therefore, for patients with few or no recent exacerbations during follow-up, an individual understanding of the longer term clinical history is necessary to decide whether to de-escalate ICS treatment. This information is essential to determine whether patients are on the right effective treatment or might have been over-treated.

\section{Scenario 2}

The GOLD ABCD classification during follow-up may change over time in an individual patient due to worsening (or sometimes improvement) of clinical characteristics; whether and how to modify pharmacological treatment in these circumstances may be unclear.

Cohort studies have shown that patients can switch between GOLD 2011 groups from year to year, including both improvement and worsening [6], but the extent to which patients change groups over time using the GOLD 2017 classification (which does not include forced expiratory volume in $1 \mathrm{~s}$ ) is not known. Reasons for clinical improvement are multiple and may include a positive response to the pharmacological treatment prescribed, the effects of non-pharmacological management (e.g. pulmonary rehabilitation and smoking cessation), improvement of comorbidities, and/or the natural resolution of a disease component (e.g. a specific airway infection). Worsening may also be attributable to a multitude of reasons, including disease progression, continued smoking, poor adherence with therapy, poor inhalation technique and the development or worsening of comorbidities. Hence, it is imperative that the clinician specifically evaluates the cause(s) of "worsening" (or improvement) before escalating (or de-escalating) pharmacological treatment specific for COPD symptoms and/or exacerbations. In patients whose pulmonary disease has deteriorated despite appropriately prescribed and used treatment, reclassification according to the GOLD ABCD assessment would lead to escalation and hence optimisation of COPD pharmacological management. In contrast, reclassification of patients who improve over time is more complex, as some will have done so as a positive treatment response which should not lead to the withdrawal or de-escalation of the successful pharmacological treatment. A useful analogy would be normalisation of blood pressure in a hypertensive patient with treatment.

\section{Scenario 3}

A highly debated point is treatment de-escalation by withdrawing ICS from patients who have not recently experienced an exacerbation [7, 8].

In this scenario, it is important to try to determine from the clinical history whether the initial use of ICS was appropriate or not and whether there was a positive treatment response. The potential for ICS side-effects should also be considered, as the risk varies between individuals. For example, pneumonia is more common in patients who are older, have lower forced expiratory volume in $1 \mathrm{~s}$, lower body mass index and a previous history of pneumonia [9]. As we progress towards precision medicine in COPD [4, 10], using blood eosinophil counts to predict the potential for ICS benefit versus risk as part of an individual approach may provide some information for decision making. There is evidence from clinical trials that higher blood eosinophil counts predict a greater ICS response [11-13]. However, a robust cut-off value for the use of this potential biomarker in clinical practice has yet to be established. 


\section{Potential alternatives}

To address these three scenarios (and others that a physician may potentially encounter in clinical practice), an individualised approach that carefully considers the clinical history, including treatment response, is of paramount importance. However, during follow-up, it is common that the detailed clinical records of an individual patient are incomplete, sparse or even missing. In such cases, what other potential options can the clinician consider?

One alternative would be to simply apply the GOLD ABCD assessment tool at this point and follow the pharmacological treatment algorithm. However, for patients already on regular maintenance treatment(s), if the latter has been successful then the ABCD classification may mislead the clinician to view the patient as over-treated, as discussed above.

A second option (possibly more theoretical than realistic), would be to stop COPD treatment and observe the patient over a period of time, and then restart the $\mathrm{ABCD}$ assessment and treatment process. This approach may be impractical/unethical for symptomatic patients unwilling to have maintenance inhaled therapy stopped for a period of time and, importantly, clinical safety has to be considered.

A third alternative for patients already on COPD treatment would be to use the same GOLD recommendations for escalation/de-escalation without the need to categorise patients into the $\mathrm{ABCD}$ groups during follow-up but rather consider "which treatable traits does this patient have?" A principle of GOLD is that treatment escalation should be decided on the need to prevent exacerbations and/or alleviate COPD symptoms such as dyspnoea [2]. These are the two key "treatable traits" [4, 5] in COPD patients and these can/should be assessed and managed separately. Indeed, the pharmacological treatment algorithm for GOLD group D already provides a template that can be easily adapted to encompass all COPD patients who are currently treated and in follow-up, as well as provide an individualised approach based on the need to treat symptoms and/or exacerbations, without the need to use the $\mathrm{ABCD}$ classification (figure 1). This treatment pathway allows escalation to occur based on additional long-acting bronchodilator treatment for symptoms and/or exacerbations (escalation arrows labelled 2 in figure 1), while additional anti-inflammatory treatment, including ICS, is only indicated to prevent exacerbations (escalation arrows labelled 1 in figure 1). There is strong evidence from randomised clinical trials for most of these recommendations; dual bronchodilators have demonstrated greater effects on symptoms and exacerbations compared to long-acting bronchodilator monotherapies [14-16], while the step up from double to triple combination treatments is supported by recent clinical trials showing effects on exacerbations and symptoms $[17,18]$. There is plenty of evidence for the step up from long-acting $\beta$-agonist (LABA) to ICS/LABA on exacerbations and quality of life [19], but the benefits of step up from long-acting muscarinic antagonist to ICS/LABA are less clear [10]. These clinical trials present group mean data, but clinicians in real-life settings deal with variations in individual patient responses. Figure 1 provides escalation and de-escalation steps to encompass individual response patterns.

Any escalation of treatment should be accompanied by a follow-up assessment of the clinical response. De-escalation to the previous treatment or switching (arrow labelled 3 in figure 1) should be considered in cases where there is no discernible response. For patients who are currently receiving treatment, the

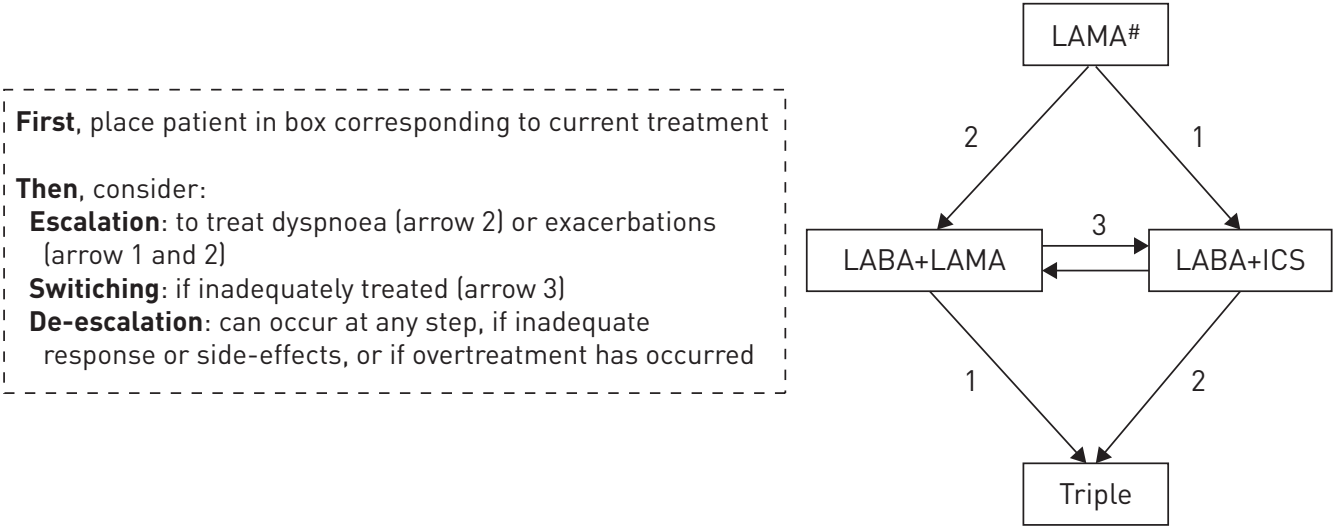

FIGURE 1 Proposed algorithm to guide pharmacological treatment in chronic obstructive pulmonary disease patients during follow-up. First, place patient in box corresponding to current treatment. Treatment escalation steps are denoted as "1" or "2"; the clinical response should be evaluated. De-escalation of treatment should be considered if there is an inadequate response or side-effects. Switching treatments (" 3 ") can be considered instead of escalation for dyspnoea or exacerbations. LAMA: long-acting muscarinic antagonist; LABA: long-acting $\beta$-agonist; ICS: inhaled corticosteroid; triple: ICS+LABA+LAMA. \# : or LABA monotherapy. 
clinician needs to consider whether the treatment level is appropriate or whether the patient is over-treated. In this context, a highly debated issue is whether to de-escalate from "triple therapy" (using two long-acting bronchodilators plus ICS) to dual bronchodilator treatment. ICS have the potential for side-effects including osteoporosis and pneumonia [2, 10]. In our view, the clinician should carefully weigh the risk of these adverse events against the potential benefits on an individual basis, using both the clinical history of exacerbations and response to ICS and, possibly, the blood eosinophil count [12]; a true precision medicine approach $[10,20]$.

Figure 1 focuses on the use of inhaled treatments during follow-up. Roflumilast, an oral phosphodiesterase type- 4 inhibitor, reduces exacerbation rates in patients with chronic bronchitis and a previous history of exacerbations [21,22], and may be considered as an add-on treatment to long-acting bronchodilator therapy with or without ICS. Azithromycin has both anti-bacterial and anti-inflammatory effects, and reduces exacerbations in patients at high exacerbation risk $[23,24]$. The benefit of this macrolide antibiotic seems to be higher in ex-smokers [25], but further characterisation of the patient subgroup most likely to respond would help clinicians, as there are concerns about antibiotic resistance during long-term use [10]. GOLD recommends the use of roflumilast or azithromycin as add-on treatments to triple therapy [2], but these treatments could also be used with long-acting bronchodilators [22], such as in cases where ICS have been ineffective.

\section{Conclusions}

The optimal pharmacological treatment of COPD should be personalised, both at initial diagnosis and during follow-up. The former can be easily accomplished using the proposed GOLD ABCD algorithm, which stratifies patients into four phenotypic groups [2]. During follow-up, however, a more individualised approach that carefully considers the response to initial therapy and the trajectory of the clinical course is needed to inform on treatment decisions. In this regard, we highlight problems that can arise especially when the previous history of patients taking maintenance treatment is not fully known or reviewed. Importantly, simply assigning patients in follow-up to one of the GOLD ACBD categories without detailed knowledge of the previous treatment history may lead to incorrect decision making, as it will be unclear whether patients categorised to groups A and B have been properly treated or over-treated according to the GOLD algorithm. Hence, we propose here that, in order to provide the best pharmacological management during follow-up for individual patients already on treatment, there is no need to formally categorise them using the ABCD grouping during follow-up. Rather, we propose a simpler algorithm (figure 1) to enable pharmacological treatment changes based on the current need to manage two of the main treatable traits of COPD, namely recurring exacerbations and/or dyspnoea. Needless to say that other treatable traits should also be considered and treated as needed $[4,5]$.

In summary, we propose that using the GOLD ABCD system remains appropriate to guide initial and subsequent pharmacological treatment in those untreated at initial assessment. However, for patients already receiving treatment, a strategy based on the individual assessment of treatable traits is likely to avoid confusion and provide a more personalised, effective and safe approach.

Acknowledgement: While the authors are members of the GOLD science committee, this article is not part of the GOLD report. The views expressed here are the personal opinions of the authors.

Conflict of interest: D. Singh reports personal fees from Apellis, Genentech, Peptinnovate and Skyepharma, as well as grants and personal fees from AstraZeneca, Boehringer Ingleheim, Chiesi, Cipla, GlaxoSmithKline, Glenmark, Menarini, Merck, Mundipharma, Novartis, Pfizer, Pulmatrix, Teva, Therevance and Verona, outside the submitted work. P. Barnes reports grants and personal fees from AstraZeneca and Boehringer Ingelheim, personal fees from Novartis, Chiesi, Menarini and Teva, and grants from Heptaris, during the conduct of the study. C. Vogelmeier reports personal fees from Almirall, Cipla, Berlin Chemie/Menarini, CSL Behring and Teva, and grants and personal fees from AstraZeneca, and Boehringer Ingelheim, Chiesi, GlaxoSmithKline, Grifols, Mundipharma, Novartis and Takeda. He has also received grants from German Federal Ministry of Education and Research (BMBF) Competence Network Asthma and COPD (ASCONET), Bayer Schering Pharma AG, MSD and Pfizer, outside the submitted work. A. Agusti reports grants and personal fees from AstraZeneca and Menarini, and personal fees from Chiesi, TEVA and Novartis, outside the submitted work.

\section{References}

1 Agusti A, Calverley P, Celli B, et al. Characterisation of COPD heterogeneity in the ECLIPSE cohort. Respir Res 2010; 11: 122-136

2 Vogelmeier CF, Criner GJ, Martinez FJ, et al. Global Strategy for the Diagnosis, Management, and Prevention of Chronic Obstructive Lung Disease 2017 Report: GOLD Executive Summary. Eur Respir J 2017; 49: 1700214.

3 Han MK, Agusti A, Calverley PM, et al. Chronic obstructive pulmonary disease phenotypes: the future of COPD. Am J Respir Crit Care Med 2010; 182: 598-604.

4 Agusti A, Bafadhel M, Beasley $\mathrm{R}$, et al. Precision medicine in airway diseases: moving to clinical practice. Eur Respir J 2017; 50: 1701655. 
5 Agusti A, Bel E, Thomas M, et al. Treatable traits: toward precision medicine of chronic airway diseases. Eur Respir J 2016; 47: 410-419.

6 Agusti A, Edwards L, Celli B, et al. Characteristics, stability and outcomes of the GOLD 2011 COPD groups in the ECLIPSE cohort. Eur Respir J 2013; 42: 636-646.

7 Magnussen H, Disse B, Rodriguez-Roisin R, et al. Withdrawal of inhaled glucocorticoids and exacerbations of COPD. N Engl J Med 2014; 371: 1285-1294.

8 Watz H, Tetzlaff $\mathrm{K}$, Wouters EF, et al. Blood eosinophil count and exacerbations in severe chronic obstructive pulmonary disease after withdrawal of inhaled corticosteroids: a post-hoc analysis of the WISDOM trial. Lancet Respir Med 2016; 4: 390-398.

9 Crim C, Dransfield MT, Bourbeau J, et al. Pneumonia risk with inhaled fluticasone furoate and vilanterol compared with vilanterol alone in patients with COPD. Ann Am Thorac Soc 2015; 12: 27-34.

10 Singh D, Roche N, Halpin D, et al. Current controversies in the pharmacological treatment of chronic obstructive pulmonary disease. Am J Respir Crit Care Med 2016; 194: 541-549.

11 Pavord ID, Lettis S, Anzueto A, et al. Blood eosinophil count and pneumonia risk in patients with chronic obstructive pulmonary disease: a patient-level meta-analysis. Lancet Respir Med 2016; 4: 731-741.

12 Singh D. Predicting corticosteroid response in chronic obstructive pulmonary disease. blood eosinophils gain momentum. Am J Respir Crit Care Med 2017; 196: 1098-1100.

13 Bafadhel M, Pavord ID, Russell REK. Eosinophils in COPD: just another biomarker? Lancet Respir Med 2017; 5: 747-759.

14 Singh D, Ferguson GT, Bolitschek J, et al. Tiotropium + olodaterol shows clinically meaningful improvements in quality of life. Respir Med 2015; 109: 1312-1319.

15 Bateman ED, Chapman KR, Singh D, et al. Aclidinium bromide and formoterol fumarate as a fixed-dose combination in COPD: pooled analysis of symptoms and exacerbations from two six-month, multicentre, randomised studies (ACLIFORM and AUGMENT). Respir Res 2015; 16: 92.

16 Wedzicha JA, Decramer M, Ficker JH, et al. Analysis of chronic obstructive pulmonary disease exacerbations with the dual bronchodilator QVA149 compared with glycopyrronium and tiotropium (SPARK): a randomised, double-blind, parallel-group study. Lancet Respir Med 2013; 1: 199-209.

17 Singh D, Papi A, Corradi M, et al. Single inhaler triple therapy versus inhaled corticosteroid plus long-acting beta2-agonist therapy for chronic obstructive pulmonary disease (TRILOGY): a double-blind, parallel group, randomised controlled trial. Lancet 2016; 388: 963-973.

18 Papi A, Vestbo J, Fabbri L, et al. Extrafine inhaled triple therapy versus dual bronchodilator therapy in chronic obstructive pulmonary disease (TRIBUTE): a double-blind, parallel group, randomised controlled trial. Lancet 2018; 391: 1076-1084.

19 Singh D, Corradi M, Spinola M, et al. Extrafine beclometasone diproprionate/formoterol fumarate: a review of its effects in chronic obstructive pulmonary disease. NPJ Prim Care Respir Med 2016; $26: 16030$.

20 Jameson JL, Longo DL. Precision medicine - personalized, problematic, and promising. N Engl J Med 2015; 372 : 2229-2234.

21 Martinez FJ, Calverley PM, Goehring UM, et al. Effect of roflumilast on exacerbations in patients with severe chronic obstructive pulmonary disease uncontrolled by combination therapy (REACT): a multicentre randomised controlled trial. Lancet 2015; 385: 857-866.

22 Calverley PM, Rabe KF, Goehring UM, et al. Roflumilast in symptomatic chronic obstructive pulmonary disease: two randomised clinical trials. Lancet 2009; 374: 685-694.

23 Albert RK, Connett J, Bailey WC, et al. Azithromycin for prevention of exacerbations of COPD. N Engl J Med 2011; 365: 689-698.

24 Uzun S, Djamin RS, Kluytmans JA, et al. Azithromycin maintenance treatment in patients with frequent exacerbations of chronic obstructive pulmonary disease (COLUMBUS): a randomised, double-blind, placebo-controlled trial. Lancet Respir Med 2014; 2: 361-368.

25 Han MK, Tayob N, Murray S, et al. Predictors of chronic obstructive pulmonary disease exacerbation reduction in response to daily azithromycin therapy. Am J Respir Crit Care Med 2014; 189: 1503-1508. 